\title{
Integrative analysis of the common genetic characteristics in ovarian cancer stem cells sorted by multiple approaches
}

Xiaoxiao Zhang ${ }^{1 \dagger}$, Yue Su${ }^{1 \dagger}$, Xue Wu${ }^{1}$, Rourou Xiao ${ }^{1}$, Yifan Wu², Bin Yang ${ }^{1}$, Zhen Wang ${ }^{1}$, Lili Guo ${ }^{1}$, Xiaoyan Kang ${ }^{1}$ and Changyu Wang ${ }^{1 *}$ (i)

\begin{abstract}
Background: Ovarian cancer is the second fatal malignancy of the female reproductive system. Based on the cancer stem cell (CSC) theory, its poor prognosis of ovarian cancer attributed to tumor recurrence caused by CSCs. A variety of cell surface-specific markers have been employed to identify ovarian cancer stem cells (OCSCs). In this study, we attempted to explore the common feature in ovarian cancer stem cells sorted by multiple approaches.
\end{abstract}

Methods: We collected the gene expression profiles of OCSCs were from 5 public cohorts and employed R software and Bioconductor packages to establish differently expressed genes (DEGs) between OCSCS and parental cells. We extracted the integrated DEGs by protein-protein interaction (PPI) network construction and explored potential treatment by the Cellminer database.

Results: We identified and integrated the DEGs of OCSCS sorted by multiple isolation approaches. Besides, we identified OCSCS share characteristics in the lipid metabolism and extracellular matrix changes. Moreover, we obtained 16 co-expressed core genes, such as FOXQ1, MMP7, AQP5, RBM47, ETV4, NPW, SUSD2, SFRP2, IDO1, ANPEP, CXCR4, SCNN1A, SPP1 and IFI27 (upregulated) and SERPINE1, DUSP1, CD40, and IL6 (downregulated). Through correlation analysis, we screened out ten potential drugs to target the core genes.

Conclusion: Based on the comprehensive analysis of the genomic datasets with different sorting methods of OCSCs, we figured out the common driving genes to regulating OCSC and obtained ten new potential therapies for eliminating ovarian cancer stem cells. Hence, the findings of our study might have potential clinical significance.

Keywords: Bioinformatic analysis, Ovarian cancer, Ovarian cancer stem cells (OCSCs), Cancer stem cells markers, Differentially expressed genes (DEGs), Therapeutic targets

\footnotetext{
* Correspondence: tjwcy66@163.com

${ }^{+}$Xiaoxiao Zhang and Yue Su contributed equally to this work.

'Department of Gynecology and Obstetrics, Tongji Hospital, Tongji Medical

College, Huazhong University of Science and Technology, 1095 Jiefang Anv., Wuhan 430030, Hubei, China

Full list of author information is available at the end of the article
}

C C The Author(s). 2020 Open Access This article is licensed under a Creative Commons Attribution 4.0 International License, which permits use, sharing, adaptation, distribution and reproduction in any medium or format, as long as you give appropriate credit to the original author(s) and the source, provide a link to the Creative Commons licence, and indicate if changes were made. The images or other third party material in this article are included in the article's Creative Commons licence, unless indicated otherwise in a credit line to the material. If material is not included in the article's Creative Commons licence and your intended use is not permitted by statutory regulation or exceeds the permitted use, you will need to obtain permission directly from the copyright holder. To view a copy of this licence, visit http://creativecommons.org/licenses/by/4.0/. The Creative Commons Public Domain Dedication waiver (http://creativecommons.org/publicdomain/zero/1.0/) applies to the data made available in this article, unless otherwise stated in a credit line to the data. 


\section{Background}

Ovarian cancer is the second most lethal gynecologic malignancy in women around the world [1]. Debulking surgery and platinum-based chemotherapy results in complete response in $70 \%$ of patients, most will relapse or even succumb to chemoresistance [2]. Significant progress in maintenance therapy has been seen by combination with poly (ADP-ribose) polymerase inhibitors, which have been approved in disease recurrence and a first-line setting among women with BRCA1/BRCA2 mutations [1]. Tumor recurrence has been attributed to suboptimal resection and the presence of residual chemo-resistant OCSCs $[2,3]$.

Over the years, multiple biomarkers have been identified exclusively or co-expressed in OCSCs and have been explored for their unique functions in tumorigenesis [4]. Several studies have contributed to the isolation and identification of OCSCs. Spheroids' formation in cancer stem cell culture has been recognized as the first commonly used approach [5, 6]. With the Hoechst 33342 dye, Side population (SP), have overexpressed several members of $\mathrm{ABC}$ transporters and exhibited some characteristics of CSCs, are collected [7, 8]. Based on cell surface markers, CD44, CD117, and CD133 etc., OCSCs have been successfully identified and isolated [9-11]. The activity of ALDH1 has been widely used in the identification of stem/progenitor cells or CSCs. Cells expressing high levels of ALDH1 can be identified by ALDEFLUOR assay and isolated by the ALDH1 antibody $[10,12,13]$.

CSCs have generally been attributed to the heterogeneity of tumors. Stem cell-associated heterogeneity resulted from intrinsic tumor plasticity can be shaped by the microenvironment [14]. Many abnormal signaling pathways of CSC play a vital role in its maintenance, survival and metastasis, including Hedgehog, Notch and Wnt $/ \beta$-catenin pathways, carcinogenic cascades such as PI3K/AKT, TGF- $\beta$, EGFR, JAK/STAT or NF- $\mathrm{kB}$ as well as transcriptional regulators such as OCT4, Nanog, YAP/TAZ and Myc [3]. OCSCs identified by the different approach has shown different mechanisms for maintaining cancer stem-like properties. They may share the same biomarkers as well as biological characteristics. This has led to an increasing interest in elucidating the underlying mechanism of OCSCs identified by different methods.

In this study, we downloaded five original microarray datasets, namely, GSE82305 [13], GSE28799 [5], GSE53759 [15], GSE94358 [16], and GSE33874 [17], from the GEO database, and these datasets contain a total of 45 samples, including 21 OCSCs samples and 24 parental cancer cell samples. We used R language software to standardize all the datasets and to get DEGs.
The 'RobustRankAggreg' package was subsequently used to integrate the results and obtain integrated differentially expressed genes (DEGs). By function and pathway analysis, we identified OCSCs share characteristics in the lipid metabolism and extracellular matrix changes. Combined with WGCNA and PPI network, we identified the hub genes of OCSC and obtained $16 \mathrm{co}-$ expressed core genes, such as FOXQ1, MMP7, AQP5, RBM47, ETV4, NPW, SUSD2, SFRP2, IDO1, ANPEP, CXCR4, SCNN1A, SPP1 and IFI27 (upregulated) and SERPINE1, DUSP1, CD40, and IL6 (downregulated). Based on the comprehensive analysis of the genomic datasets with different markers of OCSCs, we figured out the common driving signal pathways to regulating OCSCs. Finally, we obtained ten new potential therapies for the elimination of ovarian cancer stem cells.

\section{Methods}

\section{Data procession}

The gene expression profiles of OCSCs (GSE82305, GSE28799, GSE53759, GSE94358) were downloaded from the Gene Expression Omnibus (GEO) database (https:// www.ncbi.nlm.nih.gov/geo/). The dataset information is shown in Table 1. Data adjustments included data filtering, normalization, and ID transformation. Each dataset was then normalized using the normalize Between Arrays function in the R package 'limma' (http://www.bioconductor.org/). The 288 OV RNA-seq transcriptome data were download from the UCSC Xena (https://xenabrowser.net/ ) and were calculated as $\log 2(\mathrm{x}+1)$ transformed RSEM normalized counts. The 88 normal ovarian samples were obtained through the Genotype-Tissue Expression (GTEx) [18] and were calculated as $\log 2(x+1)$ transformed RSEM normalized counts.

\section{Integration of microarray data}

The $\mathrm{R}$ package 'limma' was used to test DEGs in each dataset. Genes with an adjusted $P$-value $<0.05$ and $\mid \log$ fold change $(\mathrm{FC}) \mid>1$ were considered DEGs. The DEGs in the four datasets were integrated using the $\mathrm{R}$ package 'RobustRankAggreg' (http://www.bioconductor.org/). The integrated upregulated and downregulated DEG lists were saved for subsequent analysis.

\section{Weighted gene co-expression network analysis}

The variant genes in the GSE33874 dataset were constructed to an approximate scalefree fundamental gene co-expression network using the $\mathrm{R}$ package 'WGCNA' [19]. Genes with a high correlation were clustered and the network modules were generated using the topological overlap measure (TOM). The color bars correspond to the clusters of genes can be seen as the gene module. The threshold of the coexpression module was set as $p<0.05$. 
Table 1 Details of datasets of OCSCs in the GEO

\begin{tabular}{llll}
\hline GEO accession & Platform & Organism & Details \\
\hline GSE82305 & GPL10558 & Homo sapiens & SKOV3 aldefluor(+)/aldefluor(-) \\
GSE53759 & GPL6244 & Homo sapiens & IGROV-1 spheroids/non-spheroids \\
GSE28799 & GPL570 & Homo sapiens & OVCAR3 spheroids/adherent \\
GSE94358 & GPL570 & Homo sapiens & spheroids/adherent \\
GSE33874 & GPL570 & Homo sapiens & Side population/main population \\
\hline
\end{tabular}

\section{Function and pathway analysis}

The gene ontology (GO) annotation and Kyoto Encyclopedia of Genes and Genomes (KEGG) pathway enrichment analyses of the integrated DEGs were performed using the DAVID 6.8 database (https://david. ncifcrf.gov/). GO terms were classified in three categories: biological process (BP), cellular component (CC), and molecular function (MF). The term with highest $-\log 10 \mathrm{qV}$ alue was determined the most significant enrichment. Q-values below $0.05 \quad(\mathrm{q}<0.05)$ were considered significant. The visualization of the GO and KEGG pathway enrichment analyses was performed using R 3.6.3 software.

\section{Protein-protein interaction (PPI) network construction and hub gene selection}

The PPI network of the integrated DEGs was analyzed with the STRING database (http://string-db. org/) and visualized using Cytoscape 3.8.0 software. The plug-in molecular complex detection of Cytoscape was subsequently applied to construct the subnetwork for further analysis. The top 3 cluster, with the default parameters "false Degree Cutoff $=2$ ", "Node Score Cutoff $=0.2$ ", "K-Core $=2$ " and "Max.Depth from Seed $=100$ ", was saved and listed in Table 3. Genes in a significant module of WGCNA were analyzed, and the top 3 subnets were listed in Table 2.

\section{Gene expression in immune subtypes}

A new immune classification of solid tumors has identified six immune subtypes (C1-C6) [20]. Our study population included all OV patients from TCGA with available information on Immune immune subtypes $(N=234)$. Gene expression was calculated as $\log 2(\mathrm{x}+1)$ transformed RSEM normalized counts.

\section{The prediction of potential drug based on drug-gene correlation}

DTP NIC-60 z scores and corresponding RNA-seq composite expression were downloaded from the Cellminer database (https://discover.nci.nih.gov/cellminer/ loadDownload.do) [21, 22]. Drug z-score correlated with gene expression and statistically significant $(P<$ 0.05 ) were saved and listed in Table 4 . The details of the predicted drug were listed in Table 4. Drug information was derived from the Drugbank database (https://www.drugbank.ca/).

\section{Results}

Flow chart for the study design

In this study, we conducted a comprehensive analysis of common essential genes in OCSCs isolated by deferent methods and their critical roles in OV by several computational methods. The study design was illustrated in Fig. 1.

\section{The DEGs among GSE82305, GSE28799, GSE53759 and GSE94358}

The OCSCs datasets GSE82305, GSE28799, GSE53759 and GSE94358 were normalized. The normalization of GSE82305 was shown in Fig. $2 \mathrm{~A}$ and B. The DEGs were selected using the $\mathrm{R}$ package 'limma' (adjusted

Table 2 The top 3 subnets of genes in the blue module

\begin{tabular}{lllll}
\hline Cluster & Score (Density*\#Nodes) & Nodes & Edges & Node IDs \\
\hline 1 & 21 & 21 & 210 & $\begin{array}{l}\text { WSB1, SPSB1, RNF14, MYLIP, RNF114, RNF19B, UBE2D1, KLHL21, FBXL7, SMURF2, NEDD4L, } \\
\text { GLMN, UBE2L6, ANAPC4, RNF19A, HERC2, WWP1, KBTBD7, UBE2F, TRIM32, RNF144B }\end{array}$ \\
2 & 14.174 & 24 & 163 & $\begin{array}{l}\text { IL18, CSF2, PTGER3, GNB4, GNG11, ICAM1, OXGR1, ITGAM, CSF3, ACKR3, CCR1, IL6R, CXCL3, } \\
\text { HTR1D, TLR2, C3, IL1R1, CXCL1, SSTR2, ADRA2B, BDKRB1, VCAM1, IL15, CD44 }\end{array}$ \\
3 & 11.234 & 264 & $\begin{array}{l}\text { NR4A1, TRAF1, ATF3, NFKB1, TNFAIP3, CCK, NFKBIA, IKBKB, CD83, TNFRSF10B, SERPINB2, IL6, } \\
\text { ADRB2, AVPR1B, IRAK2, AGTR1, GADD45B, KITLG, P2RY1, HRH1, FOSB, RIPK1, WNT5A, REL, } \\
\text { BTG2, STAM, JUN, RIPK2, STON1, PIK3R3, NR4A2, DUSP1, LGALS3, PACSIN2, VAMP2, CD40, } \\
\text { DNAJC6, ZFP36, TSLP, IRF1, LRP2, PIK3R1, SERPINE1, JUNB, FOSL1, SELE, NECAP2, TFRC }\end{array}$ \\
\hline
\end{tabular}




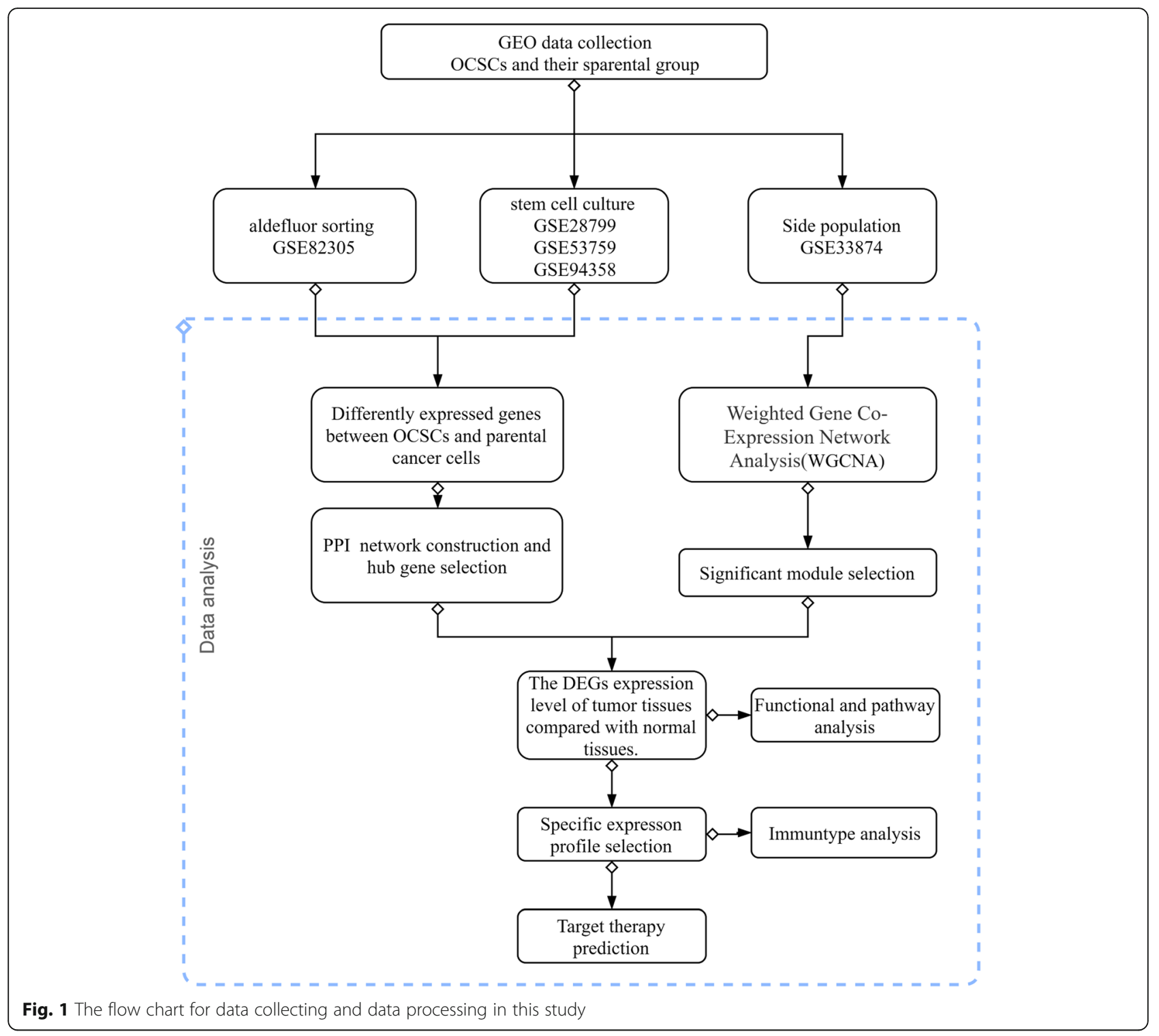

$P<0.05$ and $\mid \log$ fold change $(\mathrm{FC}) \mid>1$ ). The GSE82305 dataset contained 1474 differentially expressed genes, including 724 upregulated genes and 750 downregulated genes (Fig. 2 c). The heatmap of the top 100 genes is shown (Fig. 2 d). The GSE28799 dataset contained 1865 differentially expressed genes, including 959 upregulated genes and 936 downregulated genes (Fig. S1A-B). The GSE53759 dataset contained 273 differentially expressed genes, including 133 downregulated expression genes and 140 downregulated expression genes (Fig. S2A-B). Besides, the GSE94358 dataset contained 305 differential genes, including 50 upregulated genes and 255 downregulated genes (Fig. S3A-B).

\section{Construction of co-expression networks and identification} of key modules

We employed the WGCNA to analyze the differentially expressed genes between side population (SP) and main population (MP), which were isolated from fresh ascites obtained from 10 women with highgrade serous ovarian adenocarcinoma. The 21,655 genes in 20 samples of the GSE33874 dataset were used to construct the co-expression module. The cluster analysis on these samples and the results were depicted (Fig. S4A). Then, we screened out the softthresholding power (Fig. 3 a). When the power value was equal to 16 , the independence degree was up to 0.9 . Therefore, the power value was used to construct 


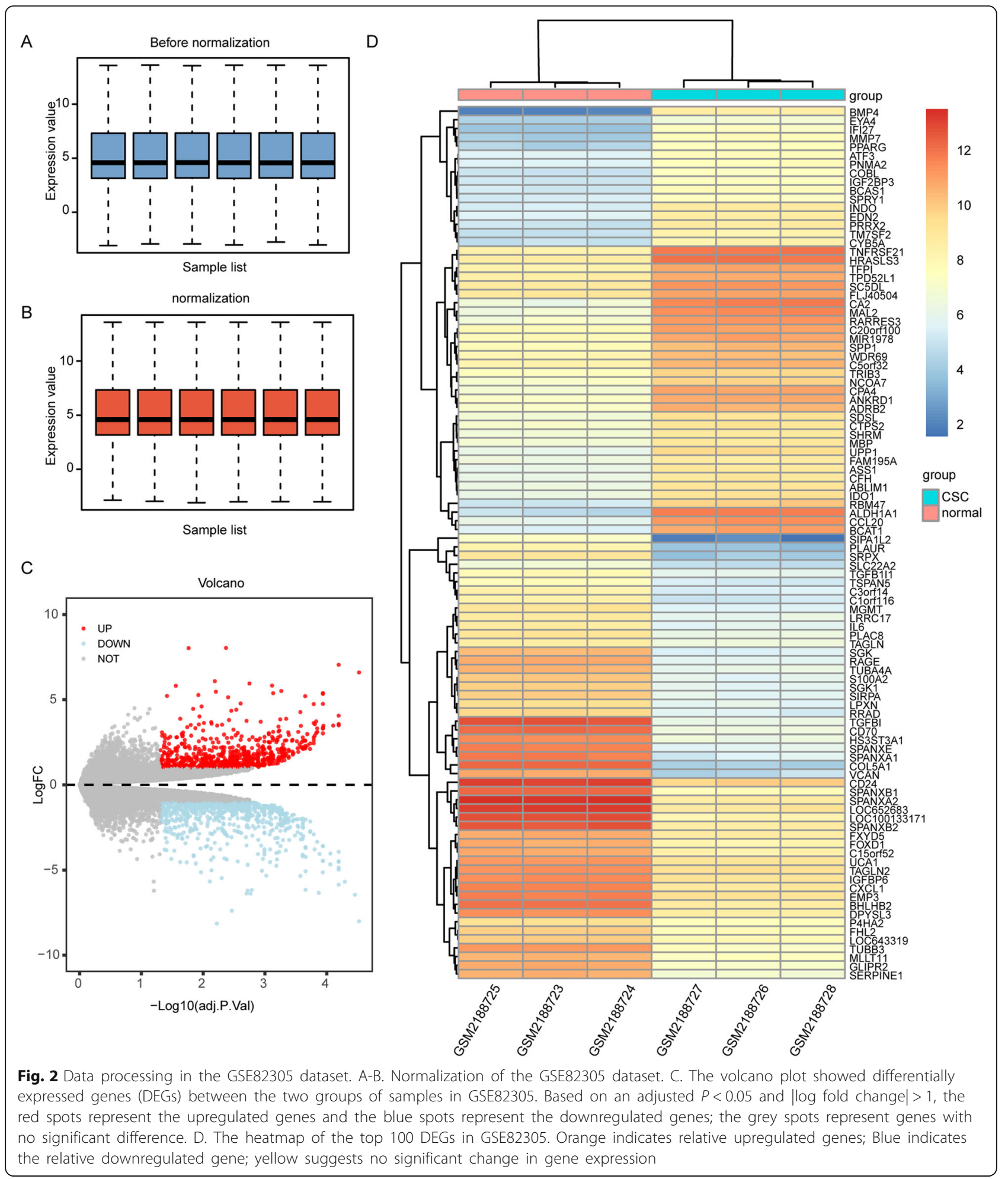

the co-expression module, and the results showed that 18 distinct gene co-expression modules were identified (Fig. 3 b). We analyzed the correlation between module eigengene and group traits and found only one co-expression module significantly correlated with SP and MP (Fig. 3 c). 1154 genes in the blue module correlated negative with SP. We performed PPI network analysis with genes in the blue module. 
A
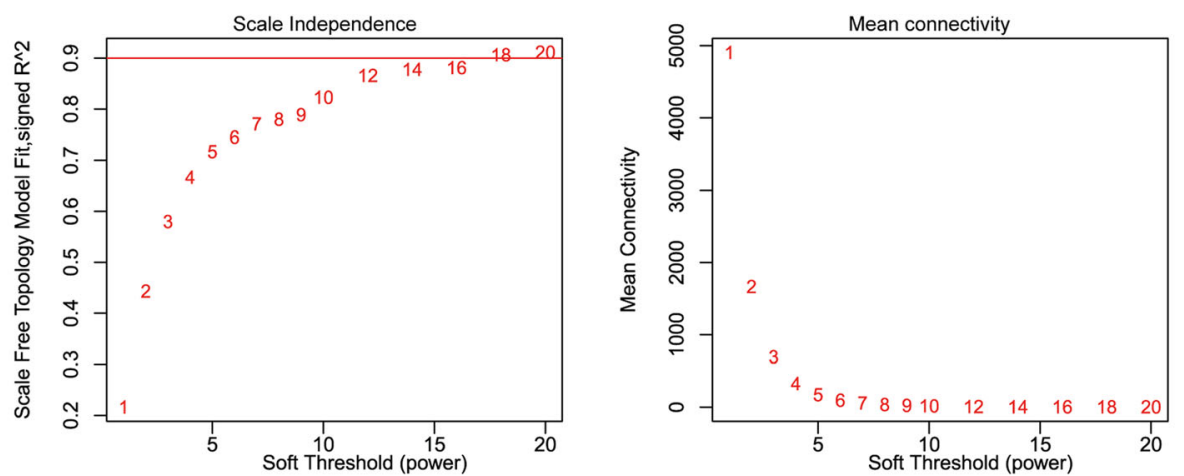

B

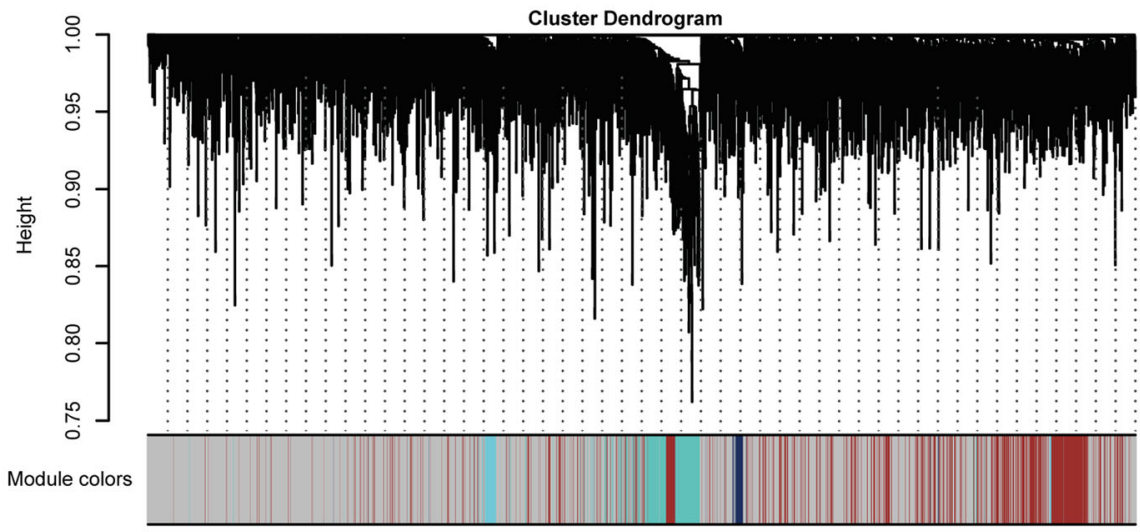

C

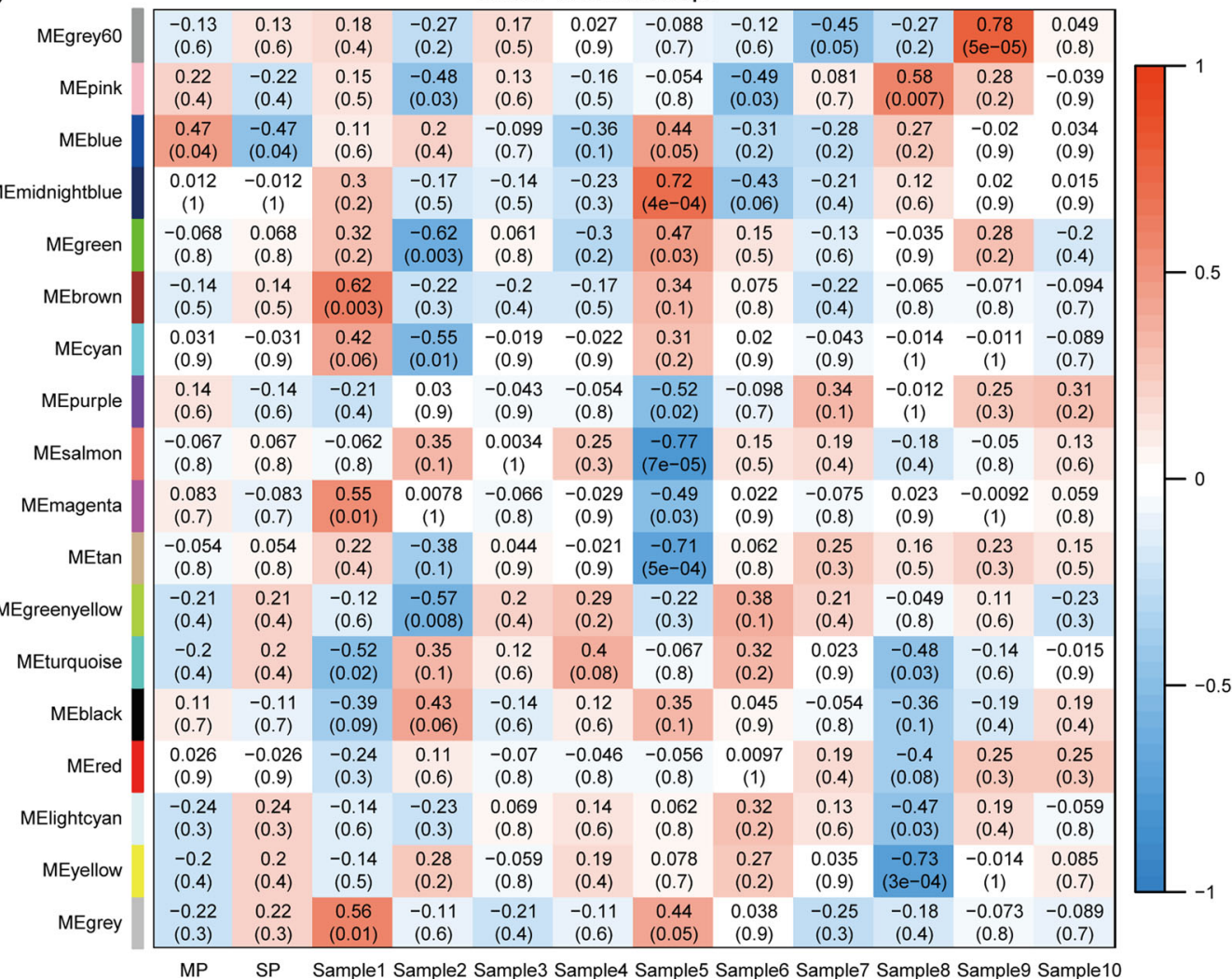

Fig. 3 WGCNA for GSE33874. A. Determination of soft-thresholding power in the WGCNA. B. Cluster dendrogram and module assignment for modules from WGCNA. C. Module-sample association relationships. Each row corresponds to a module, labeled by the same color as in (B). The correlation coefficient and $p$-value between the module and the sample or group are shown at the row-column intersection 


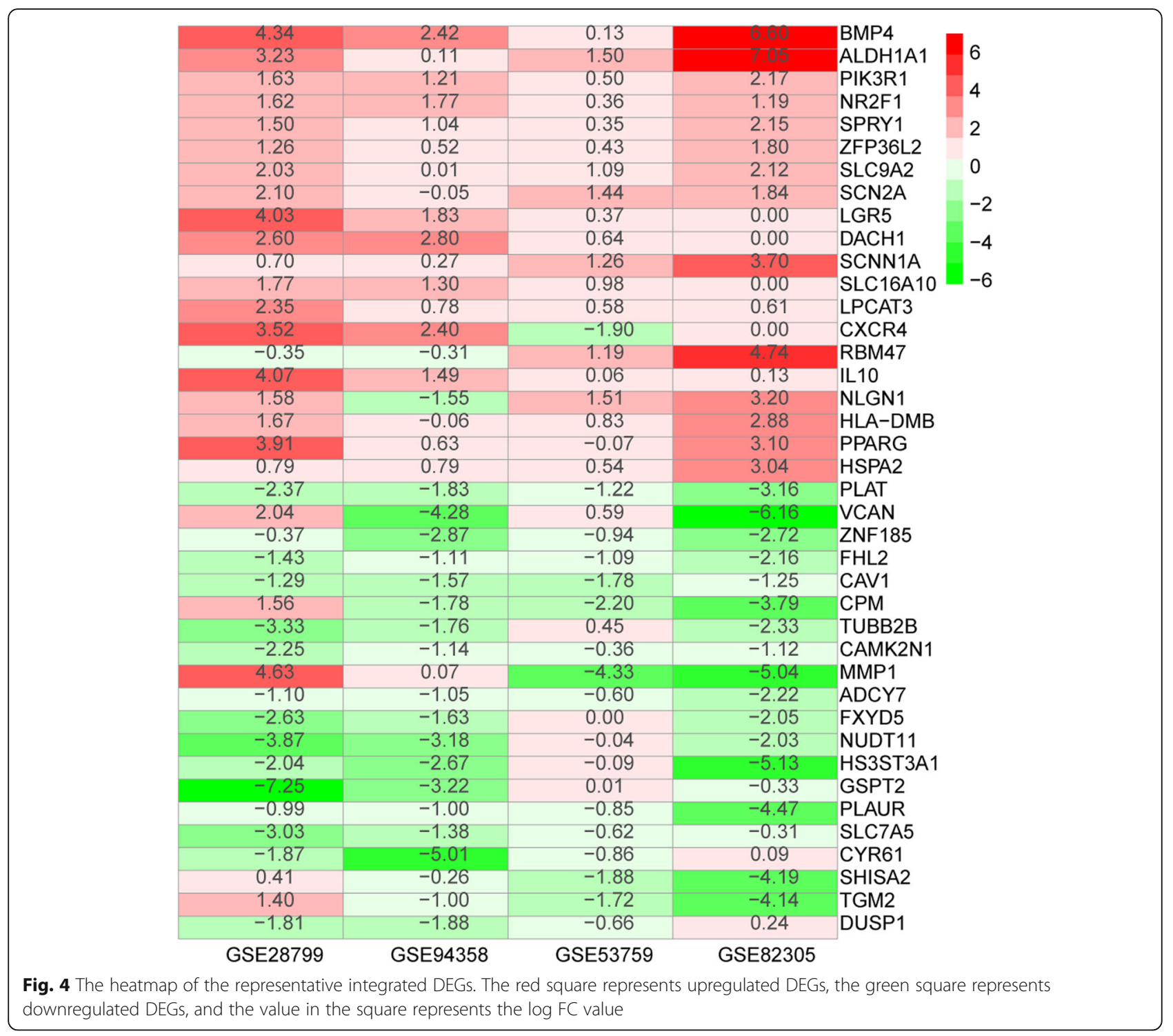

The whole network and the top 3 subnets were depicted (Fig. S4B-C), and its details were listed in Table 2. Subsequently, these genes in the blue module were subjected to GO and KEGG analysis. The top GO term and KEGG pathway in the three subnets were listed in Table S1.

\section{The integrated DEGs and their function and pathway analysis}

The DEGs of GSE82305, GSE28799, GSE53759, and GSE94358 datasets were screened using the $\mathrm{R}$ package 'limma' and sorted according to log FC. The integrated DEGs were obtained using the $\mathrm{R}$ package 'RobustRankAggreg' $(P<0.05,|\log \mathrm{FC}|>1)$. The 343 integrated DEGs, consisting of 111 upregulated genes and 232 downregulated genes, were identified (Table S2). Heat map showing the top 20 upregulated and 20 downregulated genes in the integrated DEGs (Fig. 4). Moreover, these upregulated genes and downregulated genes were subjected to cluster Profiler for GO and KEGG analysis, respectively. GO term annotation showed that these upregulated genes correlated with the regulation of lipid metabolic process, response to steroid hormone, cellular ketone metabolic process, cellular response to fatty acid, regulation of fatty acid oxidation, positive regulation of fatty acid oxidation (BP) (Fig. 5 a). GO analysis also showed that these down-regulated genes related to extracellular structure organization and extracellular matrix organization (Fig. 5b). We figured out that the upregulated genes were mainly enriched in lipid 


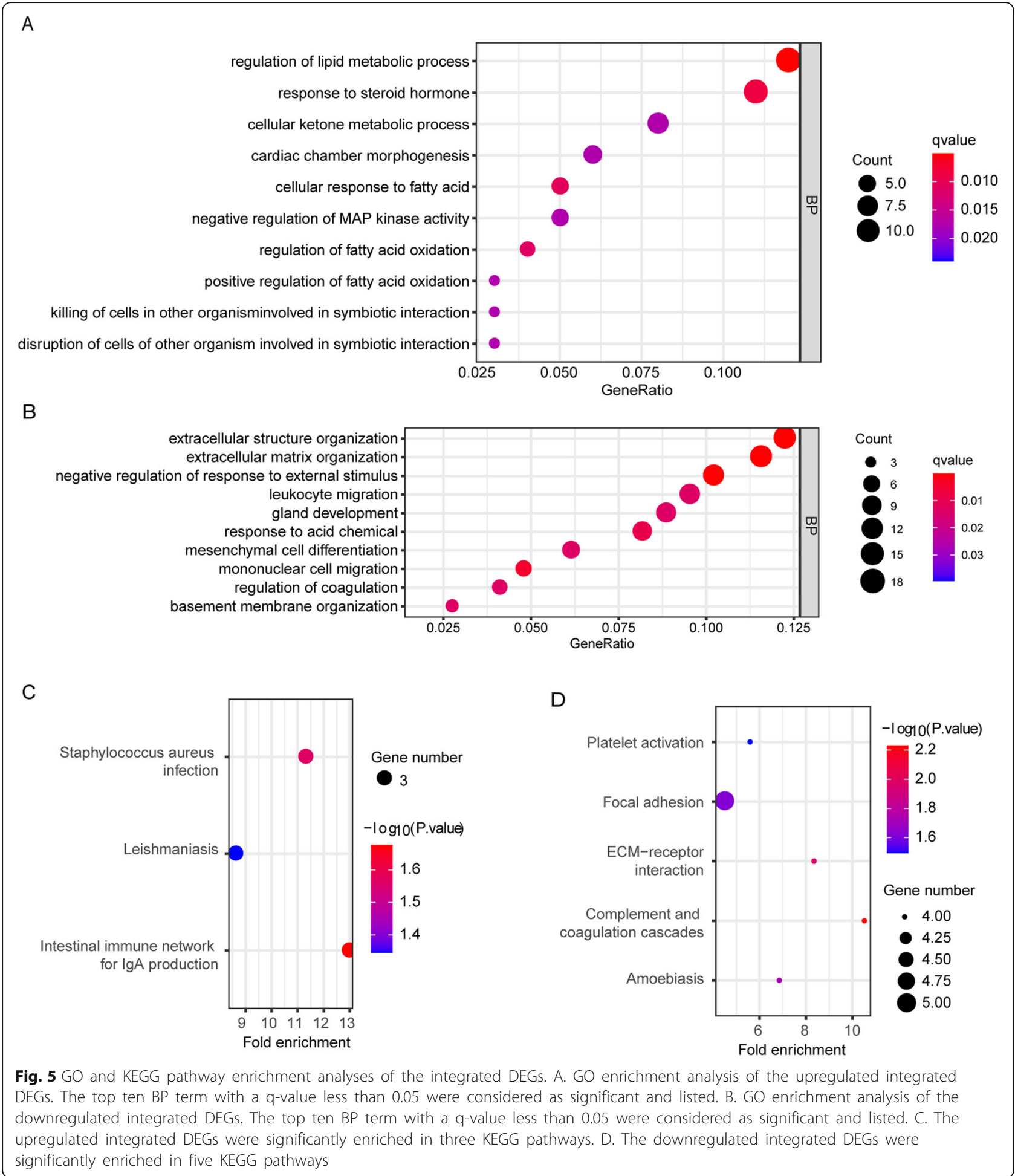

metabolism and the downregulated genes were mainly enriched in extracellular stroma. This indicated that the extracellular matrix regulated cancer stem cell behavior and character to some extent. The upregulated integrated DEGs were mainly enriched in the intestinal immune network for IgA production (Fig. 5c), and downregulated genes in focal adhesion pathways (Fig. $5 \mathrm{~d})$. We performed PPI analysis with integrated DEGs. The top 3 subnets were depicted (Fig. 6a), and its details were listed in Table 3. 
A

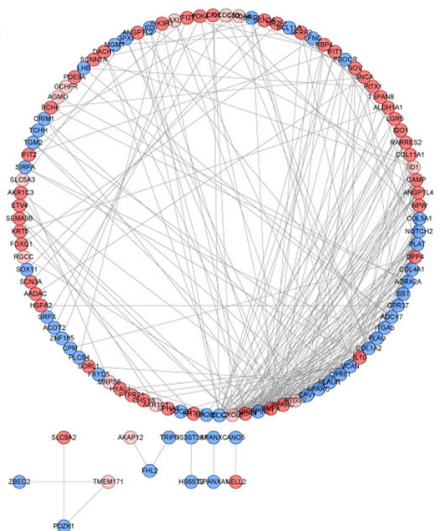

B
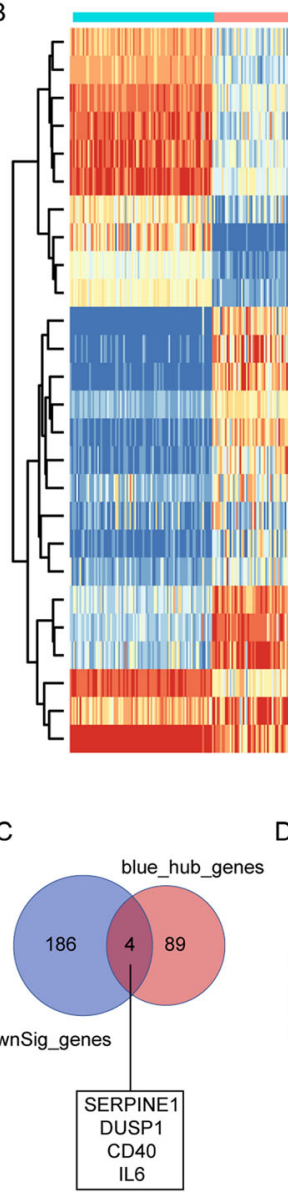
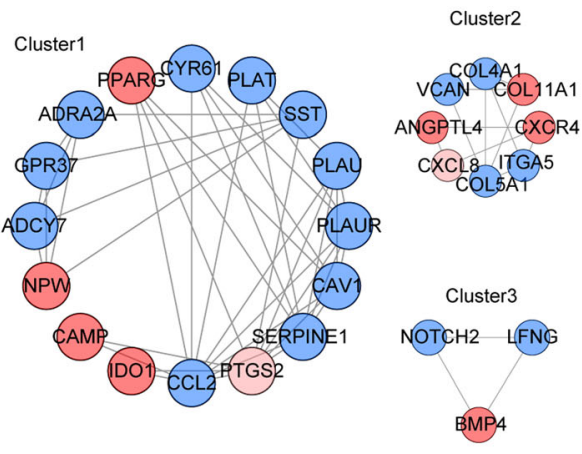

\section{Type}

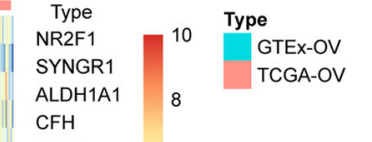

$\begin{array}{ll}\text { PDK4 } & 6\end{array}$

CYB5A

TSPAN8

CABPB 4

PPARG

SNCA 2

ГFOXQ17

MMP7

AQP5

RBM47

ETV4

NPW

SUSD2

SFRP2

IDO1

$\mid$ CXCR4

SCNN1A

SPP1 1

NUPR

RARRES2
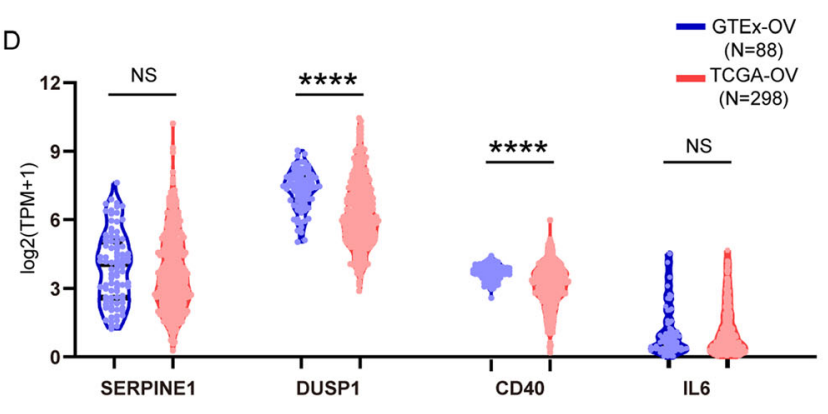

Fig. 6 PPI network construction of the integrated DEGs and SEP selection. A. The PPI network on the left was drawn using Cytoscape, and the interaction score was set to medium confidence (0.400). The network nodes represent proteins (red: upregulated proteins and blue: down-regulated proteins), and edges represent protein-protein associations. Three clusters on the right represent the top 3 subnets. B. The gene expression heatmap of the differentially expressed DEGs in TCGA and GTEx. C. Venn plot showing the intersection of the downregulated DEGs and hub genes in the blue module. D. Violin plot showing the expression of indicated genes in TCGA and GTEx (by Kruskal-Wallis test, ${ }^{*} P<0.05,{ }^{* *} P<0.01$, ${ }^{* * *} P<0.001,{ }^{* * * *} P<0.0001$ )

Expression of the integrated DEGs in the ovarian dataset of TCGA and GTEx

We tested the expression of upregulated integrated DEGs in TCGA and GTEx. Among them, 26 genes were differentially expressed (Fig. 6b). We selected the 14 genes, including FOXQ1, MMP7, AQP5, RBM47, ETV4, NPW, SUSD2, SFRP2, IDO1, ANPEP, CXCR4, SCNN1A, SPP1, and IFI27, which were both overexpressed in OV and OCSCs. Further, we used the Venn diagram to select the shared genes in upregulated integrated DEGs and the 
Table 3 The top 3 subnet of DEGs

\begin{tabular}{lllll}
\hline Cluster & Score (Density*\#Nodes) & Nodes & Edges & Node IDs \\
\hline 1 & 5.6 & 16 & 42 & ADRA2A, SST, CAV1, GPR37, PPARG, CCL2, PTGS2, CAMP, \\
2 & 3.143 & & & NPW, ADCY7, IDO1, PLAT, SERPINE1, PLAUR, PLAU, CYR61 \\
3 & 3 & 8 & 11 & COL4A1, VCAN, ITGA5, ANGPTL4, CXCR4, CXCL8, COL11A1, COL5A1 \\
\hline
\end{tabular}

genes in the blue module. We obtained four common genes, including SERPINE1, DUSP1, CD40, and IL6 (Fig. 6c), whose expression was similarly low in OV than healthy ovarian tissue (Fig. 6d). In brief, we derived specific expression profile (SEP) of OCSCs, which were composed of FOXQ1, MMP7, AQP5, RBM47, ETV4, NPW, SUSD2, SFRP2, IDO1, ANPEP, CXCR4, SCNN1A, SPP1 and IFI27 (upregulated) and SERPINE1, DUSP1, CD40, and IL6 (downregulated).

\section{SEP expression in immune subtypes}

OCSCs can survive from treatment and can be exempt from immunosurveillance. We explored whether the OCSCs signature associated with characteristics contributes to immune escape. A research group had used characteristic immune-oncologic gene signatures to cluster TCGA tumor types into six groups (C1-C6) [20]. The density of specific immune cells and overall prognosis show wide variations between the different immune subtypes. Only 4 immune subtypes were identified in OV, predominantly IFN-gamma Dominant (Immune C2, $N=138$ ) and Lymphocyte Depleted (Immune C4, N=53). C2 had the highest M1/M2 macrophage polarization, higher densities of CD8 T cells, a high proliferation rate, and the highest intratumoral heterogeneity. Therefore, we tested the expression of SEP of OCSCs in immune subtypes and found a few upregulated genes had a higher expression in the $\mathrm{C} 2$ and $\mathrm{C} 4$ group, which were characterized as lymphocyte depleted, may predict the distinct gene profile of cancer stem cells contributes to immune evasion in ovarian Cancer Patients (Fig. 7).

\section{Drug susceptibility prediction based on SEP of OCSCS}

To explore potential molecular-targeted drugs for OCSCs, we download NCI-60 drug z scores and corresponding NCI-60 cell lines RNA-seq/composite expression from the Cellminer database. The higher cell lines $\mathrm{z}$ scores have, the more sensitive to the corresponding drug they are. For better applications in the clinic, we employed the US FDA-approved drugs and drugs in clinical trials. We analyzed the correlation between drug z score and SEP. We listed four representative Pearson's correlation dot plot (Fig. 8 a). All targeted genes and relevant predicted drugs $(P<0.05)$ were shown in the Sankey diagram (Fig. 8 b). We selected drugs that were correlated with at least four target genes as a potential therapy regimen. As a result, we identified ten drugs related to SEP. Detailed information were shown in Table 4. Thus, we inferred that these drugs might be repurposed to the OCSCs with the SEP as drug targets. We extracted drugindication from DrugBank (https://www.drugbank.ca/) and listed applications of the ten medications in Table 5. For example, Ixabepilone, which was associated with CD40, CXCR4, IL6, and SERPINE1, were used in locally advanced breast cancer and metastatic breast cancer, can potentially be repurposed to treat OCSCs.

\section{Discussion}

Most ovarian cancer patients respond to initial chemotherapy, but more than $70 \%$ of patients will develop tumor recurrence and eventually develop resistance to treatment [2]. Ovarian cancer stem cells are thought to promote the recurrence of ovarian cancer and lead to the development of treatment resistance [23]. There are several methods to isolate ovarian tumor stem cells. However, the mechanisms of ovarian tumor stem cells obtained by different routes to promote tumor development are not the same. The core signaling pathways that regulate ovarian cancer stem cells remain unclear. There is still lacking in effective drugs and drug combinations to eliminate them to improve cancer survival.

In this study, the GSE82305, GSE28799, GSE53759, and GSE94358 datasets were analyzed, and 343 integrated DEGs were found. As for study GSE33874, we applied WGCNA analysis and got 18 significant enriched modules. The blue module was significantly correlated with SP and MP. The 343 integrated DEGs were then subjected to gene enrichment analysis. The upregulated genes were mainly enriched in lipid metabolism, and the downregulated genes were enriched primarily in extracellular structure organization. The KEGG pathway analysis revealed that these downregulated integrated DEGs are mostly enriched in focal adhesion, which is essential in the extracellular matrix formation. Like the metabolic characteristics of tumor cells, rapidly proliferating stem cells mainly rely on 

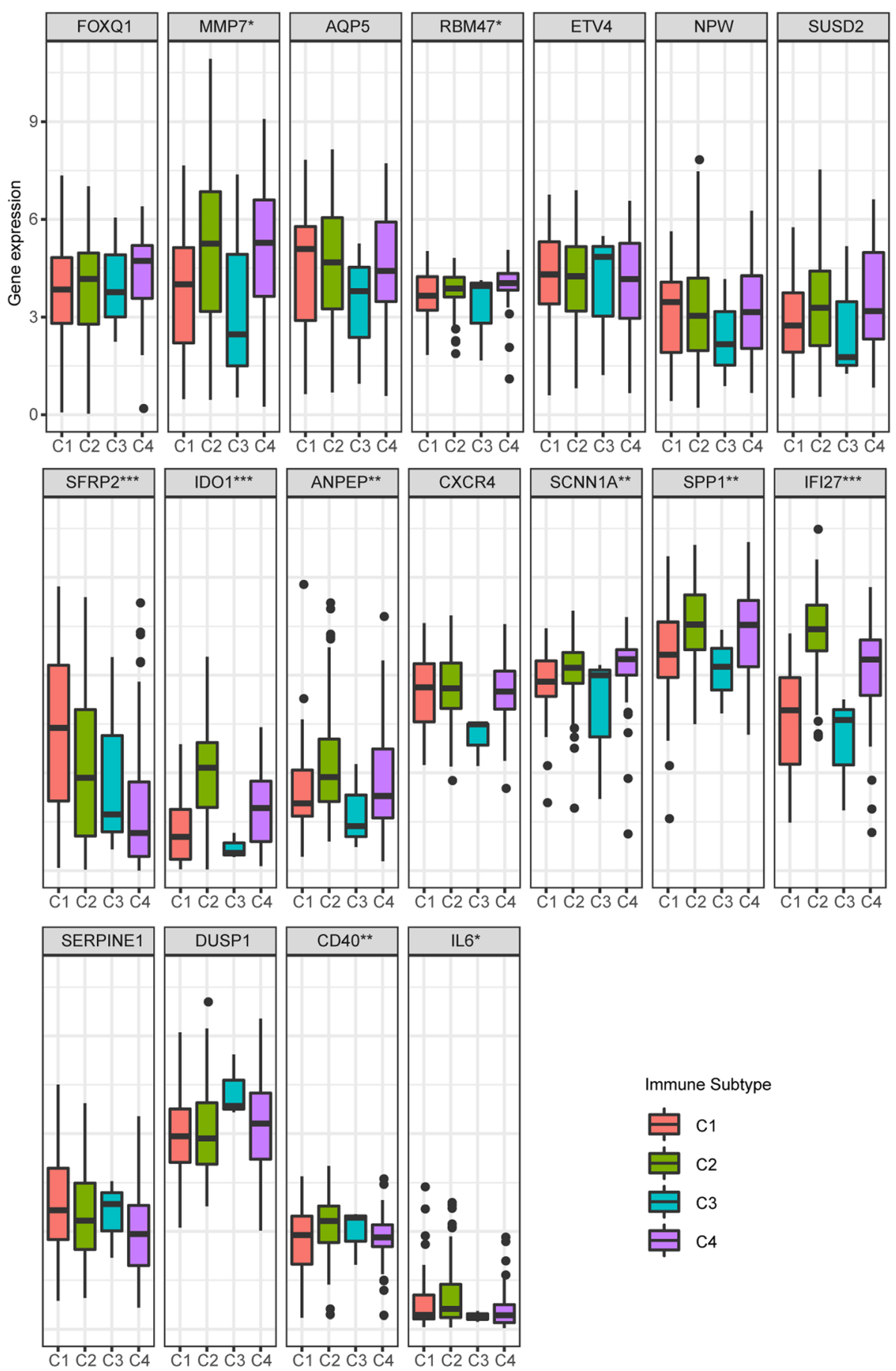

Immune Subtype

Fig. 7 Gene expression of SEP in immune subtypes. Distribution of the mRNA levels of levels for indicated genes with the most significant differences across subtypes in Ovarian cancer (by Kruskal-Wallis test, ${ }^{*} P<0.05$, ${ }^{*} P<0.01$, ${ }^{* * *} P<0.001$ )

aerobic glycolysis to provide energy [14]. In a study, chemical imaging of a single living cell was performed. They identified and described lipid unsaturation in ovarian cancer stem cells for the first time and suggested to effectively eliminate CSCs by inhibition of lipid desaturases [24]. OXPHOS pathway and lipid metabolism in cancer stem cells are recognized as targets for the development of novel anticancer therapies [25]. 

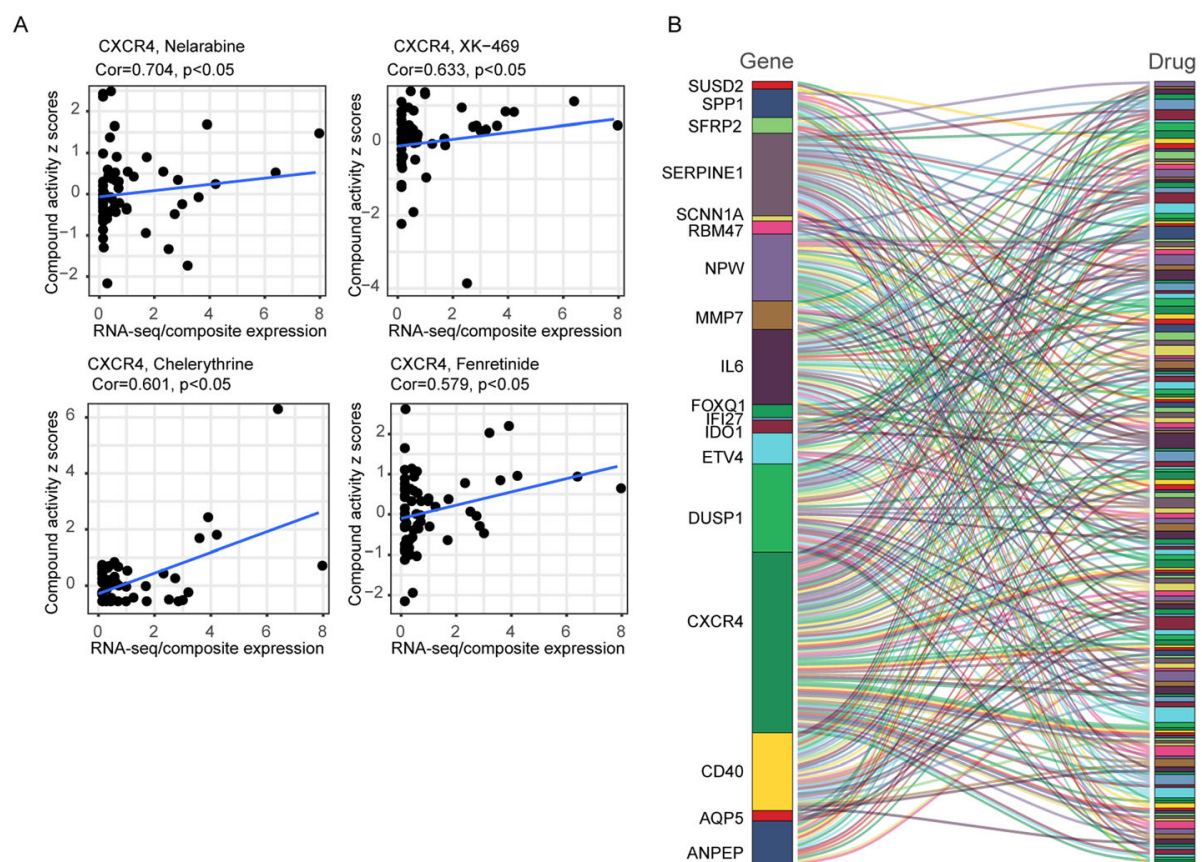

Fig. 8 Drug susceptibility prediction based on SEP. A. Representative scatter diagrams show the correlation analysis of gene expression with drug z score (by Pearson correlation test). Each black point represents an independent sample. The blue line is the linear regression. B. Sankey diagram showed the relation of targeted genes and predicted drug

In our study, we found upregulated genes were enriched in lipid metabolism, which suggested cellular event accumulation of lipids and secondary metabolites. To meet the increasing energetic requirements of CSCs, the lipid metabolic pathway can flexibly turn to the other metabolic pathways [26]. CSCs are incredibly reliant on the activity of enzymes involved in lipid metabolism, which engaged in CSCs fate decisions, such as Hippo and Wnt signal pathway [25]. Emerging evidence suggests that alterations in lipid- and fatty acidassociated pathways are essential for the maintenance of CSCs [27, 28]. Some recent evidence has demonstrated that cancer stem cell maintenance and differentiation is regulated by extracellular matrix mechanics $[29,30]$. Interactions of cells with the extracellular matrix are crucial for the establishment and maintenance of stem cell $[31,32]$. Our results also confirm that the most significant GO term in subnet3 of the blue module is response to mechanical stimulus (Table S1). In our study, we uncovered the changes in lipid metabolism and extracellular matrix are universal, independent of cell types and sorting methods. This phenomenon addresses the interactions of OCSCs with environment which result in the modulation of lipid metabolism, and thereby of OCSCs phenotype.

Studies have shown that tumor stem cells can reduce and evade from NK cells by downregulating the active ligand of NK cells, such as major histocompatibility I polypeptide related sequences A (MICA) and histocompatibility I polypeptide related sequences B (MICB), so as to escape from immune surveillance [33]. The cancer stem cells have been identified to survived within a specialized cellular niche through the crosstalk with the surrounding microenvironment [34]. Ovarian carcer was classified into four immune subtypes (C1-C4) based on the study of Thorsson et al. C2 and C4 showed poor prognosis, despite C2 had a substantial immune component. Our results showed the expression of MMP7, RBM47, and SCNN1A were significantly higher in $\mathrm{C} 2$ and $\mathrm{C} 4$ than other immune subtypes. In this regard, the complex interrelations between cancer stem cells and tumor immune microenvironment might play an vital role in MPM tumorigenesis. To identify potential drugs for OCSCs based on the SEP, we compared drug sensitivity of US FDA-approved anticancer drugs, which can be conducive to treatment. We chose ten possible drugs for OCSCs. These drugs have been used in other kinds of diseases and are believed to be further explored in tumor stem cell treatment.

In summary, the purpose of this study was to increase our understanding of the core signal pathway in OCSCs through an integrated bioinformatics analysis that aimed to identify integrated DEGs and the related pathways 
Table 4 The Person correlation of NCl-60 drug z scores and gene expression

\begin{tabular}{|c|c|c|c|}
\hline Gene & Drug & cor & $p$ value \\
\hline DUSP1 & Bafetinib & -0.43137 & 0.000579 \\
\hline ETV4 & & 0.330795 & 0.009836 \\
\hline IL6 & & -0.32127 & 0.012315 \\
\hline SERPINE1 & & -0.33833 & 0.008193 \\
\hline CXCR4 & Belinostat & 0.29389 & 0.022658 \\
\hline IL6 & & -0.26566 & 0.040218 \\
\hline NPW & & 0.39625 & 0.001724 \\
\hline SERPINE1 & & -0.29077 & 0.024203 \\
\hline DUSP1 & Cobimetinib & -0.34957 & 0.006186 \\
\hline ETV4 & & 0.417929 & 0.000892 \\
\hline IL6 & & -0.27774 & 0.031667 \\
\hline RBM47 & & 0.265132 & 0.040628 \\
\hline SERPINE1 & & -0.28517 & 0.027205 \\
\hline SPP1 & & 0.25733 & 0.047155 \\
\hline DUSP1 & Dabrafenib & -0.29456 & 0.022338 \\
\hline ETV4 & & 0.427569 & 0.000656 \\
\hline SERPINE1 & & -0.30432 & 0.018078 \\
\hline SPP1 & & 0.278976 & 0.030886 \\
\hline AQP5 & Dolastatin 10 & 0.382727 & 0.002544 \\
\hline CD40 & & -0.54851 & $5.70 \mathrm{E}-06$ \\
\hline DUSP1 & & -0.45862 & 0.000228 \\
\hline IL6 & & -0.30263 & 0.018759 \\
\hline SERPINE1 & & -0.35536 & 0.005332 \\
\hline CD40 & Ixabepilone & -0.26495 & 0.04077 \\
\hline CXCR4 & & 0.283105 & 0.028391 \\
\hline IL6 & & -0.30615 & 0.017359 \\
\hline SERPINE1 & & -0.28757 & 0.025884 \\
\hline DUSP1 & Selumetinib & -0.4288 & 0.00063 \\
\hline ETV4 & & 0.438516 & 0.000457 \\
\hline FOXQ1 & & 0.285388 & 0.027085 \\
\hline IL6 & & -0.29527 & 0.022002 \\
\hline SERPINE1 & & -0.3142 & 0.014492 \\
\hline CD40 & Tamoxifen & -0.3044 & 0.018045 \\
\hline DUSP1 & & -0.39953 & 0.001564 \\
\hline IL6 & & -0.46998 & 0.000151 \\
\hline SERPINE1 & & -0.48772 & 7.73E-05 \\
\hline CD40 & Vinorelbine & -0.48333 & $9.16 \mathrm{E}-05$ \\
\hline DUSP1 & & -0.3796 & 0.002777 \\
\hline IL6 & & -0.29874 & 0.02042 \\
\hline SERPINE1 & & -0.28779 & 0.025769 \\
\hline CXCR4 & Vorinostat & 0.421015 & 0.000809 \\
\hline IL6 & & -0.26497 & 0.040755 \\
\hline NPW & & 0.443309 & 0.000389 \\
\hline SERPINE1 & & -0.39319 & 0.001885 \\
\hline
\end{tabular}




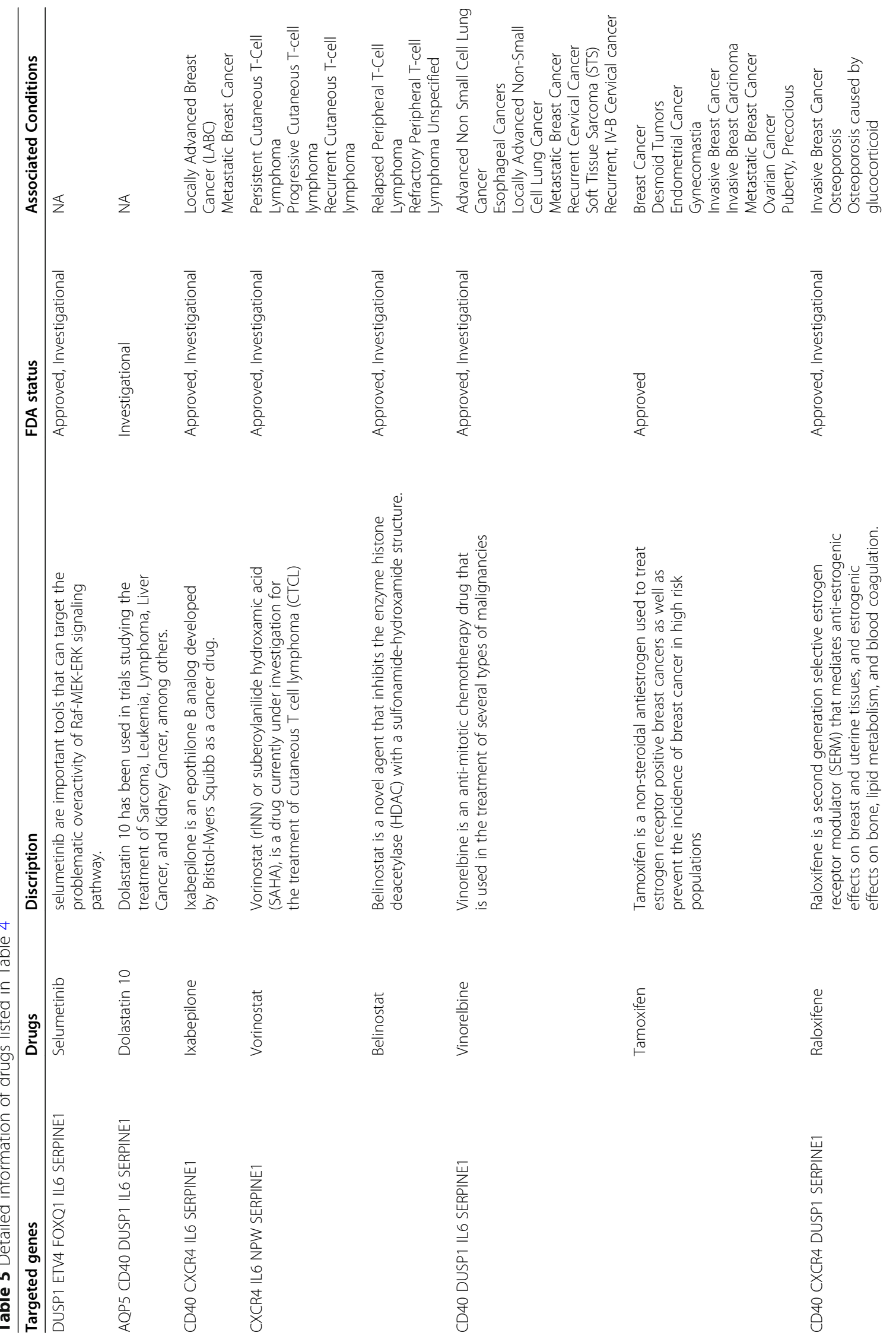




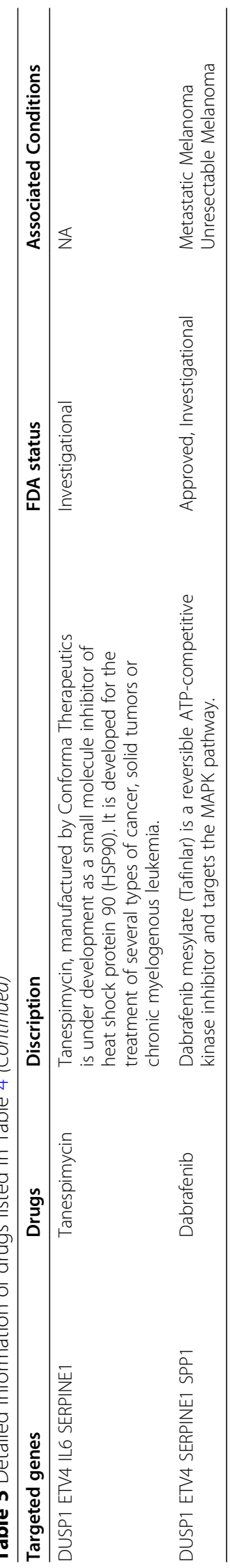


enriched in OCSCs. Our research also identified SEP that could serve as biomarkers and therapeutic targets of OCSCs. However, further research is required to establish the therapeutic efficiency of the potential drugs.

\section{Supplementary information}

Supplementary information accompanies this paper at https://doi.org/10. 1186/s13048-020-00715-7.

Additional file 1 Figure S1. Data processing in the GSE28799 dataset. A. The volcano plot showed differentially expressed genes (DEGs) between the two groups of samples in GSE28799. Based on an adjusted $P<0.05$ and |log fold change| $>1$, the red spots represent the upregulated genes and the blue spots represent the downregulated genes; the grey spots represent genes with no significant difference. B. The heatmap of the top 100 DEGs in GSE28799. Orange indicates relative upregulated genes; Blue indicates the relative downregulated gene; yellow suggests no significant change in gene expression;

Additional file 2 Figure S2. Data processing in the GSE53759 dataset. A. The volcano plot showed differentially expressed genes (DEGs) between the two groups of samples in GSE53759. Based on an adjusted $\mathrm{P}<0.05$ and |log fold change| $>1$, the red spots represent the upregulated genes and the blue spots represent the downregulated genes; the grey spots represent genes with no significant difference. B. The heatmap of the top 100 DEGs in GSE53759. Orange indicates relative upregulated genes; Blue indicates the relative downregulated gene; yellow suggests no significant change in gene expression.

Additional file 3 Figure S3. Data processing in the GSE94358 dataset. A. The volcano plot showed differentially expressed genes (DEGs) between the two groups of samples in GSE94358. Based on an adjusted $P<0.05$ and $\mid \log$ fold change $\mid>1$, the red spots represent the upregulated genes and the blue spots represent the downregulated genes; the grey spots represent genes with no significant difference. B. The heatmap of the top 100 DEGs in GSE94358. Orange indicates relative upregulated genes; Blue indicates the relative downregulated gene; yellow suggests no significant change in gene expression.

Additional file 4 Figure S4. PPI networks analysis of the blue module. A. Cluster dendrogram of 20 samples in GSE33874. B. PPI networks of genes in the blue module. $C$. The top 3 subnets of network in (B).

Additional file 5 Table S1.

Additional file 6 Table S2.

\section{Acknowledgements}

The authors thank Dr. Jianming Zeng for providing 'RobustRankAggreg' code and Dr. Ensong Guo for technical assistance.

\section{Authors' contributions}

Xiaoxiao Zhang, Yue Su, and Changyu Wang conceived the general idea. Xiaoxiao Zhang, Yifan Wu, and Xue Wu collected and analyzed the data. Yue Su, Rourou Xiao, Bin Yang, Lili Guo, Wang Zhen, Xiaoyan Kang completed figures drawing. All authors wrote the manuscript. Xiaoxiao Zhang and Yue Su should be considered joint first author. The author(s) read and approved the final manuscript.

\section{Funding}

This work was supported by grants from the National Natural and Science Foundation of China 81672580 (C. Wang), 81802612 (L. Guo).

\section{Availability of data and materials}

The datasets analyzed during the current study are available in GEO and TCGA database.

\section{Ethics approval and consent to participate}

Not applicable.

\section{Consent for publication}

Not applicable.

\section{Competing interests}

The authors have no conflicts of interest to declare.

\section{Author details}

${ }^{1}$ Department of Gynecology and Obstetrics, Tongji Hospital, Tongji Medical College, Huazhong University of Science and Technology, 1095 Jiefang Anv., Wuhan 430030, Hubei, China. ${ }^{2}$ Department of Gynecology and Obstetrics,

The Central Hospital of Wuhan, Wuhan, Hubei, China.

Received: 18 July 2020 Accepted: 10 September 2020

Published online: 25 September 2020

\section{References}

1. Lheureux S, Braunstein M, Oza AM. Epithelial ovarian cancer: Evolution of management in the era of precision medicine. CA A Cancer J Clin. 2019;4:280.

2. Ferrandina $\mathrm{G}$, Corrado $\mathrm{G}$. Treatment of platinum refractory or resistant ovarian cancer. The Lancet Oncology. 2018;19:1147-9.

3. Lupia M, Cavallaro U. Ovarian cancer stem cells: still an elusive entity? Mol Cancer. 2017;16:64.

4. Abbaszadegan MR, Bagheri V, Razavi MS, Momtazi AA, Sahebkar A, Gholamin M. Isolation, identification, and characterization of cancer stem cells: a review: Cancer stem cells: a review. J Cell Physiol. 2017;232:2008-18.

5. Wang L, Mezencev R, Bowen NJ, Matyunina LV, McDonald JF. Isolation and characterization of stem-like cells from a human ovarian cancer cell line. Mol Cell Biochem. 2012;363:257-68.

6. Zhang S, Balch C, Chan MW, Lai H-C, Matei D, Schilder JM, et al. Identification and characterization of ovarian Cancer-initiating cells from primary human tumors. Cancer Res. 2008;68:4311-20.

7. Szotek PP, Pieretti-Vanmarcke R, Masiakos PT, Dinulescu DM, Connolly D, Foster R, et al. Ovarian cancer side population defines cells with stem celllike characteristics and Mullerian inhibiting substance responsiveness. Proc Natl Acad Sci. 2006;103:11154-9.

8. Golebiewska A, Brons NHC, Bjerkvig R, Niclou SP. Critical appraisal of the side p 2011 opulation assay in stem cell and Cancer stem cell research. Cell Stem Cell. 2011:8:136-47.

9. Ffrench B, Gasch C, O'Leary JJ, Gallagher MF. Developing ovarian cancer stem cell models: laying the pipeline from discovery to clinical intervention. Mol Cancer. 2014;13:262

10. Silva IA, Bai S, McLean K, Yang K, Griffith K, Thomas D, et al. Aldehyde dehydrogenase in combination with CD133 defines Angiogenic ovarian Cancer stem cells that portend poor patient survival. Cancer Res. 2011;71:3991-4001.

11. Lupia M, Angiolini F, Bertalot G, Freddi S, Sachsenmeier KF, Chisci E, et al. CD73 regulates Stemness and epithelial-Mesenchymal transition in ovarian Cancer-initiating cells. Stem Cell Reports. 2018;10:1412-25.

12. Meng E, Mitra A, Tripathi K, Finan MA, Scalici J, McClellan S, et al. ALDH1A1 Maintains Ovarian Cancer Stem Cell-Like Properties by Altered Regulation of Cell Cycle Checkpoint and DNA Repair Network Signaling. de Bruin RAM. PLOS ONE. 2014:9:e107142.

13. Sharrow AC, Perkins B, Collector MI, Yu W, Simons BW, Jones RJ. Characterization of aldehyde dehydrogenase 1 high ovarian cancer cells: towards targeted stem cell therapy. Gynecol Oncol. 2016;142:341-8.

14. Ahmed N, Escalona R, Leung D, Chan E, Kannourakis G. Tumour microenvironment and metabolic plasticity in cancer and cancer stem cells: perspectives on metabolic and immune regulatory signatures in chemoresistant ovarian cancer stem cells. Semin Cancer Biol. 2018;53:265-81.

15. Condello S, Morgan CA, Nagdas S, Cao L, Turek J, Hurley TD, et al. $\beta$ Catenin-regulated ALDH1A1 is a target in ovarian cancer spheroids. Oncogene. 2015;34:2297-308.

16. House CD, Jordan E, Hernandez L, Ozaki M, James JM, Kim M, et al. NFkB promotes ovarian tumorigenesis via classical pathways that support proliferative Cancer cells and alternative pathways that support ALDH+ Cancer stem-like cells. Cancer Res. 2017;77:6927-40.

17. Vathipadiekal V, Saxena D, Mok SC, Hauschka PV, Ozbun L, Birrer MJ. Identification of a Potential Ovarian Cancer Stem Cell Gene Expression Profile from Advanced Stage Papillary Serous Ovarian Cancer. PLoS ONE. 2012;7:e29079.

18. The GTEx Consortium, Ardlie KG, Deluca DS, Segre AV, Sullivan TJ, Young TR, et al. The Genotype-Tissue Expression (GTEx) pilot analysis: Multitissue gene regulation in humans Science 2015;348:648-660.

19. Langfelder P, Horvath S. WGCNA: an R package for weighted correlation network analysis. BMC Bioinformatics. 2008;9:559. 
20. Thorsson V, Gibbs DL, Brown SD, Wolf D, Bortone DS, Ou Yang T-H, et al. The Immune Landscape of Cancer. Immunity. 2018;48:812-830.e14.

21. Reinhold WC, Varma S, Sunshine M, Elloumi F, Ofori-Atta K, Lee S, et al. RNA Sequencing of the NCI-60: Integration into CellMiner and CellMiner CDB. Cancer Res. 2019;79:3514-24.

22. Reinhold WC, Sunshine M, Varma S, Doroshow JH, Pommier Y. Using CellMiner 1.6 for Systems Pharmacology and Genomic Analysis of the NCl60. Clin Cancer Res. 2015;21:3841-52.

23. Garcia-Mayea Y, Mir C, Masson F, Paciucci R, LLeonart ME. Insights into new mechanisms and models of cancer stem cell multidrug resistance. Semin Cancer Biol. 2020;60:166-80.

24. Li J, Condello S, Thomes-Pepin J, Ma X, Xia Y, Hurley TD, et al. Lipid Desaturation Is a Metabolic Marker and Therapeutic Target of Ovarian Cancer Stem Cells. Cell Stem Cell. 2017;20:303-314.e5.

25. Chae YC, Kim JH. Cancer stem cell metabolism: target for cancer therapy. BMB Rep. 2018;51:319-26.

26. Ghoneum A, Gonzalez D, Abdulfattah AY, Said N. Metabolic plasticity in ovarian Cancer stem cells. Cancers. 2020;12:1267.

27. Wang T, Fahrmann JF, Lee H, Li Y-J, Tripathi SC, Yue C, et al. JAK/STAT3Regulated Fatty Acid $\beta$-Oxidation Is Critical for Breast Cancer Stem Cell SelfRenewal and Chemoresistance. Cell Metabolism. 2018;27:136-150.e5.

28. Pascual G, Avgustinova A, Mejetta S, Martín M, Castellanos A, Attolini CS-O, et al. Targeting metastasis-initiating cells through the fatty acid receptor CD36. Nature. 2017;541:41-5.

29. Qiao S, Zhao Y, Li C, Yin Y, Meng Q, Lin F-H, et al. An alginate-based platform for cancer stem cell research. Acta Biomater. 2016;37:83-92.

30. Totaro A, Zhuang Q, Panciera T, Battilana G, Azzolin L, Brumana G, et al. Cell phenotypic plasticity requires autophagic flux driven by YAP/TAZ mechanotransduction. Proc Natl Acad Sci U S A. 2019;116:17848-57.

31. Pickup MW, Mouw JK, Weaver VM. The extracellular matrix modulates the hallmarks of cancer. EMBO Rep. 2014;15:1243-53.

32. Brown Y, Hua S, Tanwar PS. Extracellular matrix-mediated regulation of cancer stem cells and chemoresistance. Int J Biochem Cell Biol. 2019;109:90-104.

33. Bruttel VS, Wischhusen J. Cancer stem cell immunology: key to understanding tumorigenesis and tumor immune escape? Front Immunol. $2014 \mathrm{Jul} ; 29: 5$.

34. Varas-Godoy M, Rice G, Illanes SE. The crosstalk between ovarian Cancer stem cell niche and the tumor microenvironment. Stem Cells Int. 2017;2017:1-8.

\section{Publisher's Note}

Springer Nature remains neutral with regard to jurisdictional claims in published maps and institutional affiliations.

Ready to submit your research? Choose BMC and benefit from:

- fast, convenient online submission

- thorough peer review by experienced researchers in your field

- rapid publication on acceptance

- support for research data, including large and complex data types

- gold Open Access which fosters wider collaboration and increased citations

- maximum visibility for your research: over $100 \mathrm{M}$ website views per year

At BMC, research is always in progress.

Learn more biomedcentral.com/submissions 